\title{
PRINCIPALES DETERMINANTES ECONÓMICOS DEL CONSUMO EN COLOMBIA 2000 - 2015
}

\author{
MAIN ECONOMIC DETERMINANTS OF CONSUMPTION IN \\ COLOMBIA 2000 - 2015
}

\section{PRINCIPAIS DETERMINANTES ECONÔMICOS DO CONSUMO NA COLÔMBIA 2000 - 2015}

\section{CABRERA VALVERDE _ Diana Gisell, ROSERO OLIVEROS _ Tania Marcela, RIASCOS_Julio César}

Egresada del Programa de Mercadeo, Universidad de Nariño. Email: dianagisell. c14@gmail.com, Colombia.

Egresada del Programa de Mercadeo, Universidad de Nariño. Email: taniamrosero@gmail.com, Colombia.

Magister en Gerencia y Asesoría Financiera, Universidad Mariana. Profesor e investigador Universidad Mariana y Universidad de Nariño. Integrante de los Grupos de Investigación Contar, Coyuntura Económica y Social, y Frontera Sur. E-mail: jriascos2009@hotmail.com, Colombia.

Recibido: 20 de noviembre de 2018 Aprobación definitiva: 13 de julio de 2019 DOI: http://dx.doi.org/10.22267/rtend.192001.109

\section{RESUMEN}

El propósito de este estudio reside en explicar las causas que han afectado el consumo en Colombia durante el periodo comprendido entre los años $2000 \mathrm{y}$ 2015, esto de acuerdo con los modelos keynesianos, ciclo de vida e ingreso permanente de Milton Friedman. 
Así, con base en la revisión de la literatura teórica y empírica, se analizaron dos variables relevantes: el ingreso a partir del PIB y la inflación tomada como el IPC, obteniendo como resultado que la variable más significativa para el estudio del consumo en Colombia es el ingreso, conclusión alcanzada mediante el uso de un modelo estadístico de aplicación multivariada no complejo.

En consecuencia, se recomienda aliviar las cargas tributarias, dinamizar los subsidios y las inversiones en educación, al tiempo que controlar la tasa de interés para propiciar mejores niveles de consumo en el país.

Palabras clave: consumo, ingreso, índice de precios al consumidor (ipc), inflación, producto interno bruto (pib), devaluación, tasa de cambio

JEL: E21, E25, E31, E23, E52

\section{ABSTRACT}

The purpose of this study is explain the casualties that have affected consumption in Colombia during the period between the 2000's and 2015's, this according to the keynesian models, life cycle and permanent entrance of Milton Friedman.

So, based on the review of the theoretical and empirical literature, two relevant variables were analyzed: inflow from GDP and inflation taken with the CPI, resulting in the most significant variable for consumption in Colombia, conclusion reached through the use of a multivariate statistical.

It is therefore advisable to relieve tax burdens, to boost subsidies and investments in education, in time to control interest rates to provide better levels of consumption in the country.

Keywords: consumption, income, consumer's price index (cpi), inflation, gross domestic product (gdp), devaluation, exchange rate

JEL: E21, E25, E31, E23, E52 


\section{RESUMO}

O propósito deste estudo é explicar as causas que afetaram o consumo na Colômbia no período de 2000 a 2015, segundo os modelos keynesianos, ciclo de vida e ingresso permanente de Milton Friedman.

Assim, com base na revisão da literatura teórica e empírica, duas variáveis relevantes foram analisadas: ingresso a partir do PIB e inflação tomadas como o IPC, obtendo como resultado que a variável mais significativa para o estudo do consumo na Colômbia é a ingresso, conclusão alcançada através do uso de um modelo estatístico multivariado não-complexo.

Consequentemente, recomenda-se aliviar as cargas tributárias, impulsionar subsídios e investimentos em educação, enquanto controla-se a taxa de juros para promover melhores níveis de consumo no país.

Palavras-Chave: consumo, ingresso, índice de preços no consumidor (ipc), inflação, produto interno bruto (pib), desvalorização, taxa de câmbio JEL: E21, E25, E31, E23, E52

\section{INTRODUCCIÓN}

El presente documento tiene como propósito mostrar los cambios que se han evidenciado en el consumo final en Colombia durante el periodo comprendido entre los años 2000 a 2015. Partiendo de que el consumo es afectado por distintas variables, el objetivo es hallar los principales determinantes que llevan una relación a largo plazo con el consumo colombiano y su incidencia. En este caso se han considerado para el análisis del estudio dos variables principales que lo explican: ingreso (PIB) e inflación (IPC t-1), razonamiento obtenido después de probar en 8 modelos, las relaciones entre el consumo y las variables: PIB, IPC, IPC t-1, Desempleo, Desempleo t-1, TCR, TCR t-1.

Partiendo del modelo keynesiano y de las cifras obtenidas a partir del Boletín de Indicadores Económicos del año 2016 del Banco de la República, se efectuó un modelo estadístico de aplicación multivariada no complejo, para determinar la incidencia que tiene el crecimiento económico y la inflación sobre los niveles 
de consumo en Colombia, encontrando que existe una relación directa del crecimiento económico frente al consumo y una relación inversa de la inflación respecto al consumo con $95 \%$ de confianza estadística. Lo que es compatible con el estudio de Clavijo y Fernández (1989) sobre el consumo privado en Colombia, así como la relación entre el consumo y el ingreso en Colombia elaborada por Carrasquilla (1989).

\section{MARCO TEÓRICO}

Según Iregui y Melo (2009) los modelos teóricos de consumo están basados en tres hipótesis principales: "Ingreso absoluto (Keynes, 1983), ingreso permanente (Friedman, 1957) y ciclo de vida (Modigliani, 1949)".

Se considera que el Consumo "es parte de la demanda agregada y depende del ingreso disponible." (Jiménez, 2006) del mismo modo, Keynes plantea en la Ley Psicológica Fundamental que ingreso y consumo tienen una relación directa, dicha ley se resume en que, en una determinada sociedad, el aumento del ingreso o renta implica que el consumo también aumente, pero en una proporción menor al incremento del ingreso mismo. Además, Keynes (1936) en su libro Teoría general de la ocupación, el interés y el dinero, afirma que el principal determinante del consumo es el ingreso, pero que existe una parte del consumo que no depende de este, implicando más variables que lo causan; sin embargo, en este modelo, variables como la tasa de interés, el dinero y la tasa de cambio no son relevantes, hecho por el cual asume la función consumo como estable y únicamente dependiente del ingreso real disponible (Keynes, 1992: 93), es decir:

$$
\mathbf{C}=\mathbf{f}(\mathbf{Y d})
$$

Según Morettini (2002), otra hipótesis de Keynes respecto a la propensión marginal a consumir PMgC es que ésta disminuya a medida que el ingreso aumente. Respondiendo a la siguiente ecuación:

$$
\mathrm{C}=\mathrm{Co}+\mathbf{c Y d}
$$

$\mathbf{C}$ es el consumo, $\mathbf{C} \mathbf{0}$ es el consumo autónomo, es decir, que no depende del ingreso, $\mathbf{c}$ es la pendiente, es decir, la $\mathrm{PMgC}$, Yd es el ingreso real disponible (Morettini, 2002: 4). 
Por otro lado, Milton Friedman en la teoría del ingreso permanente (Nobel de Economía, 1976), plantea también que el ingreso es un determinante del consumo, lo descompone en dos partes: consumo permanente y consumo temporario, debido a que existen dos tipos de ingreso. El consumo permanente es aquel en el que los consumidores no esperan variaciones a futuro, en contraste, el ingreso temporario es aquel donde el consumidor considera que no se mantendrá en el tiempo; por lo tanto, la hipótesis de Friedman consiste en suponer que el consumo depende principalmente del ingreso permanente.

Otro argumento que indica que las modificaciones en los ingresos de las personas o su disponibilidad a gastar, afectan el consumo, es el planteamiento del Banco de la República "la renta personal disponible es la cantidad de ingresos de que disponen los hogares para gastar o para ahorrar una vez pagados los impuestos y recibidas las transferencias."

Para contrastar los anteriores planteamientos teóricos de la variable Ingreso, Fisher (1993), expone que los determinantes del consumo y el ahorro, dependen de tres variables, R1, R2 y r, donde R1 y R2 son las rentas registradas en dos años, y $\mathrm{r}$ hace referencia al tipo de interés. Donde sólo dos variables son importantes: $\mathrm{W}=\mathrm{R} 1+\mathrm{R} 2 / 1+\mathrm{r}$ y $\mathrm{r}$, es decir, la riqueza y el tipo de interés:

$$
\mathbf{C}=\mathbf{f}(\mathbf{r}, \mathbf{W})
$$

Al interpretar R1 y R2 como las rentas registradas en los dos años, el consumo de cada año depende no de la renta sino de la riqueza ("Renta permanente"). Adicionalmente, definir el ahorro como la diferencia entre la renta registrada y el consumo, hace que el ahorro dependa de la renta (Fisher, 1993: 83), porque:

$$
\text { S1 }=\text { R1-C }=\text { R1-f }(\mathbf{r}, \mathbf{W})
$$

En el análisis del consumo, es pertinente tratar la teoría moderna del consumo que hace referencia a las decisiones que se toman de por vida. Inicialmente la hipótesis del ciclo vital de Franco Modigliani (Nobel de Economía, 1985) pretende explicar la aparente contradicción existente entre la teoría del consumo keynesiana y las evidencias empíricas. El postulado principal es que los consumidores intentan poseer un nivel constante de calidad de vida a lo largo de su entera existencia ante las variaciones que experimentaba la renta, en otras 
palabras, con el fin de mantener constante el nivel de consumo durante toda la vida (Situación que otorga más satisfacción que, por ejemplo, consumir más en los primeros años que en los restantes) el consumidor debe ahorrar durante su vida activa para desahorrar en la vida pasiva:

$$
\mathbf{C} * \mathbf{V T}=\mathbf{Y} * \mathbf{V A}
$$

Siendo $\mathbf{C}$ el consumo anual, $\mathbf{Y}$ el ingreso anual, VA los años de vida activa y VT los años de vida total. Se desarrolla bajo los supuestos: No existe la riqueza, no se reciben ni se dejan herencias, los precios son constantes, el ahorro no genera interés, la corriente de ingresos no se detiene, y es constante y no existe desempleo (Morettini, 2002: 10).

En tanto que la teoría de la renta permanente, se centra en la predicción del nivel de renta, del que dispone un consumidor en toda su vida. La teoría moderna del consumo "predice que un aumento temporal de impuestos (y, por tanto, una reducción temporal del ingreso disponible) tendrá muy poco efecto en el consumo y en la demanda agregada." (Dornbusch, Fischer y Startz, 2009: 319) En contraposición Dornbusch, Fischer y Startz plantean que el efecto de los impuestos sobre el consumo es insignificante, para Carlos León y María Miranda en el análisis macroeconómico para la empresa, los impuestos son realmente importantes en la determinación del consumo, teniendo que "el consumo depende del ingreso disponible, el ingreso disponible es el que perciben los agentes menos los impuestos directos. C: C + c (Y - t Y)" (León y Miranda, 2003: 44) por lo tanto, incrementar los impuestos implica que el ingreso disponible sea mucho menor, y la capacidad de compra se afecte contrayendo el mercado.

Según Carlos Otálora Urquizu (2009), coincide con la visión de León y Miranda, donde establece que el consumo privado $(\mathrm{Cp})$, depende del ingreso disponible de las personas (Yd), del nivel de precios al consumidor (IPC), la propensión media a consumir, gustos y preferencias de las personas; especifica que el impuesto es una variable que afecta directamente al consumo, "mediante instrumentos tributarios se puede alterar alguna de las variables mencionadas anteriormente, por ejemplo, de manera directa a través del impuesto a la renta de personas, se puede modificar el ingreso disponible y en consecuencia el nivel del consumo privado" (Otálora, 2009: 143). Explica que las personas de bajos 
ingresos soportan cargas tributarias mayores a las que soportan quienes tienen ingresos elevados, cita el siguiente ejemplo:

\section{Tabla 1}

Comportamiento de dos personas, persona A de bajos ingresos y persona $B$ de altos ingresos sometidas a un Impuesto al Consumo del 10\%. (Otálora, 2009:145).

\begin{tabular}{|l|c|c|}
\hline \multicolumn{1}{|c|}{ Conceptos } & Persona A & Persona B \\
\hline Ingreso anual (Y) & 5.000 & 100.000 \\
\hline Gastos en consumo & 4.900 & 45.000 \\
\hline Impuestos al consumo del 10\% (t) & 490 & 4.500 \\
\hline Carga tributaria t/Y (en \%) & 9,80 & 4,50 \\
\hline
\end{tabular}

Fuente: Elaboración propia.

Lo anterior se sustenta en que el aumento de los impuestos, es decir, la aplicación de un impuesto general a las ventas, se refleja en el aumento del nivel de precios; debido a que el aumento de impuestos va a trasladarse a los consumidores, afectando en mayor proporción a personas de bajos ingresos, que a los de ingresos altos y, por ende, disminuye el consumo.

En lo que respecta a la inflación que es la tasa de variación de los precios y, el nivel de precios es determinado por la acumulación de inflaciones anteriores (Dornbusch, Fischer y Startz, 2009:41), en razón a que la inflación modifica los precios al alza, el consumo se afecta negativamente, porque con precios altos, el incentivo a consumir decae; la relación de inflación y consumo es indirecta.

Para Clavijo y Fernández (1989), la tasa de inflación (INF) captura el denominado efecto del "ahorro forzoso" sobre el consumo; aparece el desahorro obligado, puesto que en el corto plazo los trabajadores carecen de mecanismos para defender su ingreso ante un proceso inflacionario. Por consiguiente, la inflación reduce el consumo.

Los costes de una inflación alta se distinguen con facilidad en una economía, debido a que su lubricante es el dinero; cuando los países tienen inflaciones que mensualmente duplican sus precios, el dinero deja de ser un medio útil de cambio 
y en consecuencia, la producción disminuye, dicho de otra manera, aunque "la inflación no produce una pérdida directa de producción como el desempleo" (Dornbusch, Fischer y Startz, 2009: 173), sino que es un efecto colateral que posteriormente conlleva una menor rentabilidad para las empresas, y a que sea necesario despedir empleados o reducir sus salarios, finalmente, el consumo se modifica, en este caso con una reducción.

La inflación genera incertidumbre en las decisiones económicas, debilita el nivel de ingreso, genera menores posibilidades de ventas para las empresas, mayores costos salariales, problemas de decisión frente a aspectos futuros de las personas, en contraste, una inflación baja y estable [que mejora] el bienestar de la población porque disminuye la incertidumbre, brinda confianza en los precios relativos, hay una asignación eficiente de los recursos, además de seguridad en el contenido informativo de precios futuros y existe un incentivo para invertir, dado que el grado de confianza a largo plazo es mayor (Bemanke et al, 1999).

Por su parte Dornbusch, Fischer y Startz (2009), refieren que:

La noción de que los costos de la inflación totalmente anticipada son bajos no cuadra bien con la intensa aversión a la inflación que se manifiesta en la política y las medidas públicas. El motivo más importante para esta aversión es que la experiencia inflacionaria de Estados Unidos es de inflación imperfectamente anticipada, variable, cuyos costos son muy distintos. (Dornbusch, Fischer y Startz, 2009: 167).

Según estos autores las personas se preocupan esencialmente por la inflación imperfectamente anticipada la cual implica mayores costos y alta variabilidad, y no por la inflación total.

Esto significa que la posibilidad de una inflación inesperada introduce otro elemento de riesgo. Este riesgo adicional elimina parte del atractivo de los intercambios entre empresas y consumidores. Es un costo evidente asociado con una inflación imprevista y es muy difícil medirlo (Dornbusch, Fischer y Startz, 2009: 167)

En contraste, James Tobin planteó en un discurso presidencial de la American Economic Association (1972), que un poco de inflación le hace bien a la economía 
(y reduce la tasa natural del desempleo) porque ofrece un mecanismo necesario para bajar los salarios reales sin reducir los salarios nominales.

No obstante, para Dornbusch, Fischer y Startz (2009:175) "en un mundo que cambia, algunos salarios reales tienen que subir y otros tienen que bajar para alcanzar la eficiencia económica y tener poco desempleo." Entonces, cuando la Inflación es cero, las empresas deben hacer recorte directo en los salarios nominales de sus trabajadores, pero cuando ésta tiene un valor cercano al $3 \%$ o es una inflación positiva, no requiere disminuir los salarios nominales para que el salario real se ajuste, sólo implica esperar a que la Inflación haga su efecto, es decir, en palabras del propio autor "imagine a world in which there are fixed costs of adjusting wages and inflation is positive. In this environment, inflation causes real wages to fall automatically over time." (Akerlof, Dickens y Perry, 1996: 69).

Por ende, como la inflación tiene efectos sobre los precios y la renta, que es el principal determinante del consumo, genera significativos efectos sobre el consumo en general "así, la tasa inflacionaria es de mayor importancia para prestamistas y prestatarios de largo plazo; y es todavía más importante para la vivienda." (Dornbush, Fisher y Startz, 2009: 172).

Para Dornbush, Fisher y Startz (2009), otro determinante fundamental es la tasa de cambio (Tipo de cambio), pues a través de la devaluación se explica en parte la inflación de precios de un país y el consumo de un país disminuye significativamente ante una moneda devaluada o una TRM alta, la relación con esta variable, al igual que con la inflación, es indirecta.

De acuerdo con Jiménez (2006: 133) otro determinante del consumo es la tasa de interés, en razón a que las tasas de interés altas son incentivos al ahorro "el nuevo equilibrio se encuentra con un menor consumo presente y un mayor consumo futuro, ya que el consumo futuro se hace más barato cuando la tasa de interés aumenta" (Jiménez, 2006: 133); la tasa de interés tiene una relación inversa con el consumo presente: el aumento de tasas de interés hoy genera menor consumo hoy, y relación directa con el consumo futuro: $\mathrm{Al}$ aumentar las tasas de interés hoy, mañana se producen aumentos en el consumo. El efecto ingreso no existe en el conjunto de la economía. 
Para Dornbusch, Fischer y Startz (2009), la tasa de interés "manifiesta la tasa de pago sobre un préstamo u otra inversión, además del pago del capital, expresada como porcentaje anual" (Dornbusch, Fischer y Startz, 2009: 43). Si la tasa aumenta, se debe disponer de una porción mayor de salario para cubrir la deuda, y al consumo se destina un monto menor, o sea se lo reduce; entonces la tasa de interés es un determinante de relación inversa al consumo.

El desempleo también se constituye en un factor importante a la hora de explicar el consumo según Dornbush, Fisher y Startz (2009:150), porque las personas que se encuentran en situación de desempleo perciben ingresos menores o nulos y eso dificulta su capacidad adquisitiva de bienes y servicios ofrecidos en una economía, luego la conclusión es que si la tasa de desempleo aumenta el consumo se desestimula, es decir, el desempleo tiene una relación inversa frente al consumo.

Michio Morishima (1981), plantea, siguiendo a Keynes, que cuando un trabajador está desempleado, su demanda de bienes de consumo disminuye, pero no llega a cero. Por consiguiente, la relación del desempleo frente al consumo es directa, sustentado en que "el valor total de la demanda de bienes de consumo por parte de los trabajadores es igual a la suma realmente pagada en salarios a los trabajadores empleados" (Morishima, 1981: 234).

En palabras de Morten O. Ravn (2008): la conclusión es que "surprisingly, has shown that the introduction of a labor market participation choice is of considerable analytical convenience, since it allows us to derive a very simple testable relationship between labor market tightness and consumption". (Ravn, 2008: 42).

Esto explica que el desempleo afecta el ingreso disponible de las personas y éste a su vez el consumo.

Para CEPAL (2001), otra variable que presenta relación inversa con el consumo es la pobreza, "un individuo será pobre cuando no satisfaga el consumo de bienes y servicios que le permitan vivir y desenvolverse dignamente en su contexto social. El indicador de bienestar en este caso estará dado por el consumo de bienes en específico." (CEPAL, 2001: 5), por lo tanto, si la pobreza se incrementa, el consumo se deteriora significativamente. 
La última variable que explica el consumo son los subsidios, para Echeverri (1998) constituyen un adicional al ingreso, razón que motiva el consumo, puesto que la capacidad adquisitiva de un individuo es mayor, "aplicados por la vía del gasto público, implican transferencias de unos sectores a otros mediados por una acción específica, una programación y una definición de políticas estratégicas del estado." (Echeverri, 1998: 99) En conclusión, la relación subsidios y consumo es directa.

En tal sentido, la relación de las variables desarrolladas permite explicar el consumo decreciente de la siguiente manera:

La devaluación conlleva al incremento de la inflación, lo cual aumenta los precios y disminuye la rentabilidad para las empresas, en consecuencia la tasa de desempleo crece y con ello también la pobreza, haciendo que el ingreso disponible de las personas sea menor y por ende consuman menos. Aunado a ello, si los subsidios disminuyen y se incrementan los impuestos, la cantidad de ingresos disponible para consumir se reduce. Y si las tasas de interés son altas se estimula el ahorro, haciendo que en el presente el ingreso disponible y el consumo se reduzcan, pero en el futuro se incrementarán ambos.

\section{ANTECEDENTES}

Sergio Clavijo y Javier Fernández (1989), revisan el estado actual de los determinantes del consumo privado (o del ahorro) en Colombia y depuran las estimaciones econométricas que han aportado como sustento empírico de las diferentes hipótesis.

Las estimaciones que permiten establecer que los principales determinantes del consumo privado real se explican por los efectos temporales de los términos de intercambio, que afectan el ingreso nacional real, así como efectos distributivos de las exportaciones y el efecto complementario de las importaciones.

Los autores proponen para el caso de Colombia que el consumo e ingreso a usar es el consumo privado real (CPR) y el ingreso nacional real disponible (INA): Relación (CPR/INA).

Cuando el incremento del ahorro privado real (APR) sobre INA mejora, la razón CPR/INA desciende; en consecuencia, el efecto esperado INA/PIB es negativo 
sobre la participación del consumo. Esta variable es un elemento fundamental en la Teoría del Ingreso Permanente.

Por otra parte, las exportaciones hacen que CPR/INA disminuya, mientras que las importaciones tienen un efecto positivo sobre el mismo.

Respecto a la tasa impositiva TAX/PIB, en Colombia como el esfuerzo tributario se ha centrado en impuestos indirectos: Impuesto a las ventas e impuesto al valor agregado, estos no tienen por qué afectar el consumo. Coincide con la visión de Dornbush, Fisher y Startz, que refiere también, un bajo efecto de los impuestos en el consumo, en contraposición de los planteamientos de León y Miranda, así como de Carlos Otálora, para quienes esta variable tiene implicaciones significativas en el consumo.

La tasa de dependencia demográfica (TDD), teñida como la porción de la población en edad de trabajar, tiene una relación inversa con CPR/INA. En tanto se aumente TDD se eleva la proporción del ahorro, y cuando TDD desciende por el "añejamiento poblacional" hay desahorro, pero también incremento del consumo. Concuerda con los postulados de la teoría del ciclo de vida de Modigliani.

La tasa de interés real (TIR) a través de cual es posible explorar el efecto precio, implica que la relación CPR/INA descienda al postergar el consumo ante un mejor retorno financiero para quienes elijan ahorrar hoy. Así, proposiciones como las de Jiménez e Iregui y Melo, presentan este mismo análisis.

Clavijo y Fernández finalmente concluyen que el endeudamiento externo, implica aumento del consumo y, por ende, menor proporción de la inversión; y precisan que la liberación de importaciones (Bienes de consumo) afecta negativamente la generación del ahorro doméstico.

Dentro de las estimaciones adelantadas para Colombia de la relación entre el consumo y el ingreso puede encontrarse la elaborada por Carrasquilla (1989), quien a través de una serie de regresiones concluye que la implicación estocástica de la no existencia de predictores significativos del consumo agregado, asociada a la HIPER, es violada en el caso colombiano en la medida que se atribuye cierta 
influencia del ingreso sobre el consumo. (Carrasquilla, 1989). Así, se reconoce que en Colombia el ingreso afecta al consumo y, en consecuencia, los hogares tienden a acumular bienes durables y activos como la vivienda.

Según Juan Nicolás Hernández (2006), los determinantes del consumo, son el ingreso y la edad. El consumo depende también, de variables adicionales al ingreso de estado estacionario, tales como la riqueza personal y los precios relativos.

Mediante los patrones de consumo que se pueden presentar en un ciclo particular de vida, la conducta del individuo puede variar, haciendo que no consuma todo su ingreso hasta el final de su vida, puesto que "el individuo de hecho puede heredar parte de su ingreso acumulado a las generaciones posteriores" (Bernheim, 1985). Al momento de ampliar las variables que pretenden medir el bienestar de los hogares, se han encontrado determinantes en su relación con los patrones de consumo no solo aquellas asociadas con la riqueza relativa y la estructura de edades. Para casos particulares, como en el Japón, Wakabayashi y Horioka (2005), presenta cómo las restricciones al crédito han incidido en el impacto positivo y significativo del ingreso corriente sobre el consumo en dicho país, reafirmando el efecto de las imperfecciones en el mercado de capitales sobre el consumo y su relación con el exceso de sensibilidad.

El estudio realizado por Ana María Ireguí y Ligia Alba Melo miembros de la Unidad de Investigaciones de la Gerencia Técnica del Banco de la República (2009), plantea que el principal determinante del consumo es la tasa de interés teniendo en cuenta las expectativas acerca del futuro, seguida por la riqueza (Renta permanente) por consiguiente, los Bancos Centrales podrían incidir directamente en el consumo a través de variaciones en la tasa de interés, o indirectamente por medio de cambios en el ingreso permanente.

Las tasas de interés afectan las decisiones de consumo, porque los cambios en estas se transmiten a los hogares; un aumento en las tasas de interés, reduce el valor presente del ingreso futuro de las personas, lo cual hace que el valor presente de los recursos que el consumidor obtiene a lo largo de su vida disminuya.

Concluyen que el consumo depende de la riqueza y de la tasa de interés, de ahí que, al existir una brecha entre el ingreso de los hogares y el consumo, cuando 
este es mayor al ingreso, se produce una financiación con crédito, lo que genera un ingreso nominal que por ende aumenta la demanda de bienes y servicios, que, si supera la oferta potencial, presiona al alza el nivel de precios, es decir, conlleva a la inflación.

En efecto, otra variable que contribuye a la explicación del consumo, es el crédito, ya que este lo dinamiza, aun cuando el nivel de ingreso inicialmente no lo permitiera.

\section{METODOLOGÍA}

En el análisis, el método utilizado fue un modelo estadístico sencillo desarrollado en el programa de Gretl, que se puede manejar como una aplicación multivariada no compleja, en la cual se desarrollaron ocho modelos estadísticos, utilizando variables como: Ingreso (PIB), Inflación (IPC), Inflación t-1, desempleo, desempleo $\mathrm{t}-1$, devaluación real y devaluación real $\mathrm{t}-1$, permitiendo encontrar que las variables determinantes del consumo en Colombia con mayor nivel de significancia (90\%) son el Ingreso expresado en PIB y la inflación t-1 explicadas en el modelo número seis. ${ }^{1}$ Lo anterior, basado en resultados de estudios anteriores.

NOTA: Se espera que el modelo cumpla con los supuestos: normalidad, homocedasticidad, no autocorrelación, no multicolinealidad.

Es de resaltar que en los estudios que se ejecutan sobre el consumo, se manejan modelos econométricos complejos que cumplen o satisfacen no solamente los supuestos de una modelación, sino que además, se utilizan, para predecir y determinar la significancia de las variables. En este caso, se trata de un ejercicio no complejo para mirar la relación de las variables.

El estudio verifica la teoría y únicamente refuerza los resultados de investigaciones anteriores y bajo ningún punto es un análisis que pretenda aportar nuevas evidencias de factores que expliquen el consumo.

1 La realización de modelos de consumo con variables como: desempleo, desempleo t-1, devaluación real y devaluación real t-1 no alcanzaron el nivel de significancia por encima del $90 \%$, por lo que fueron excluidas del análisis. 


\section{ANÁLISIS GRÁFICO}

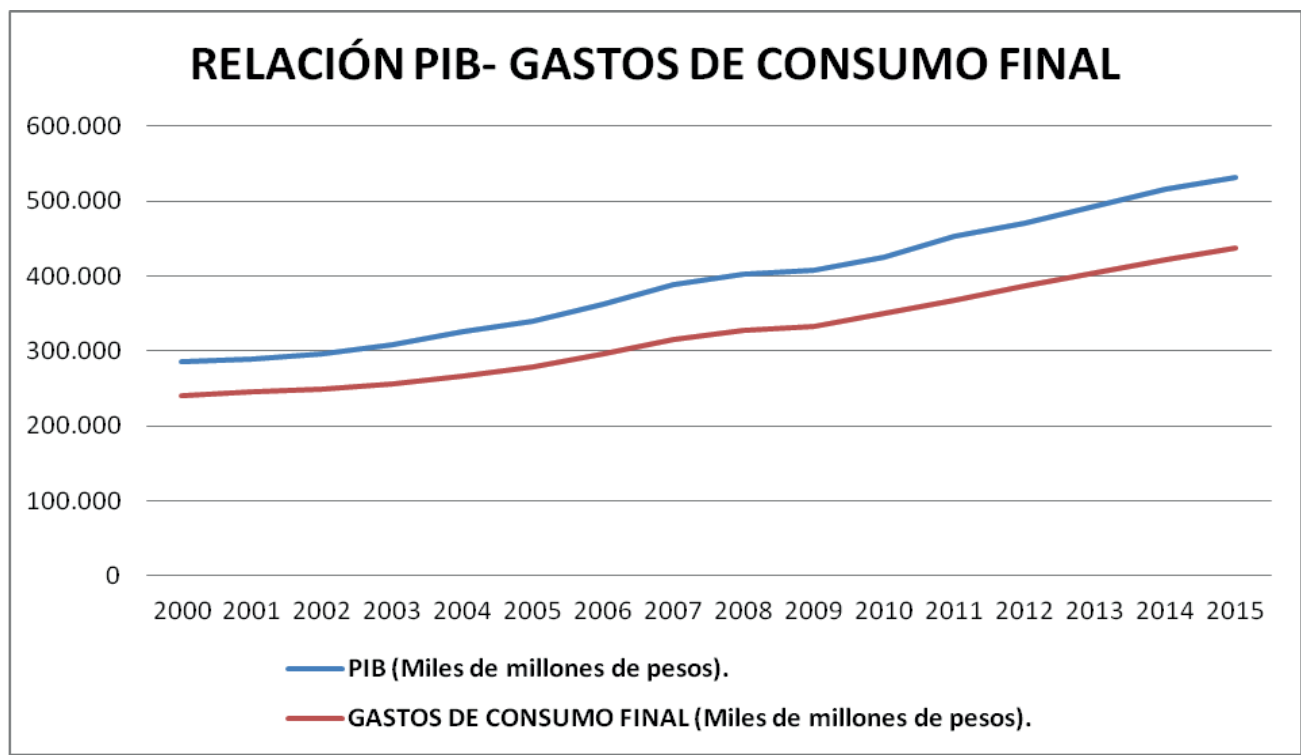

\section{Figura 1}

Relación ingresos (PIB) y gastos de consumo final colombiano (2000 - 2015).

Fuente: Boletín de Indicadores Económicos 2016, Banco de la República.

Con la Fig. 1 Se verifica la relación directa entre los Ingresos (PIB), y el consumo en Colombia en el periodo comprendido entre el año 2000 - 2015. Sustentando lo que planteaba Carrasquilla (1989). Así, cuando el ingreso aumenta el consumo también, aunque en una menor proporción, mostrando lo planteado en la "Ley psicológica fundamental" de Keynes (1992). El excedente se destina al ahorro o al pago de deudas o créditos. 


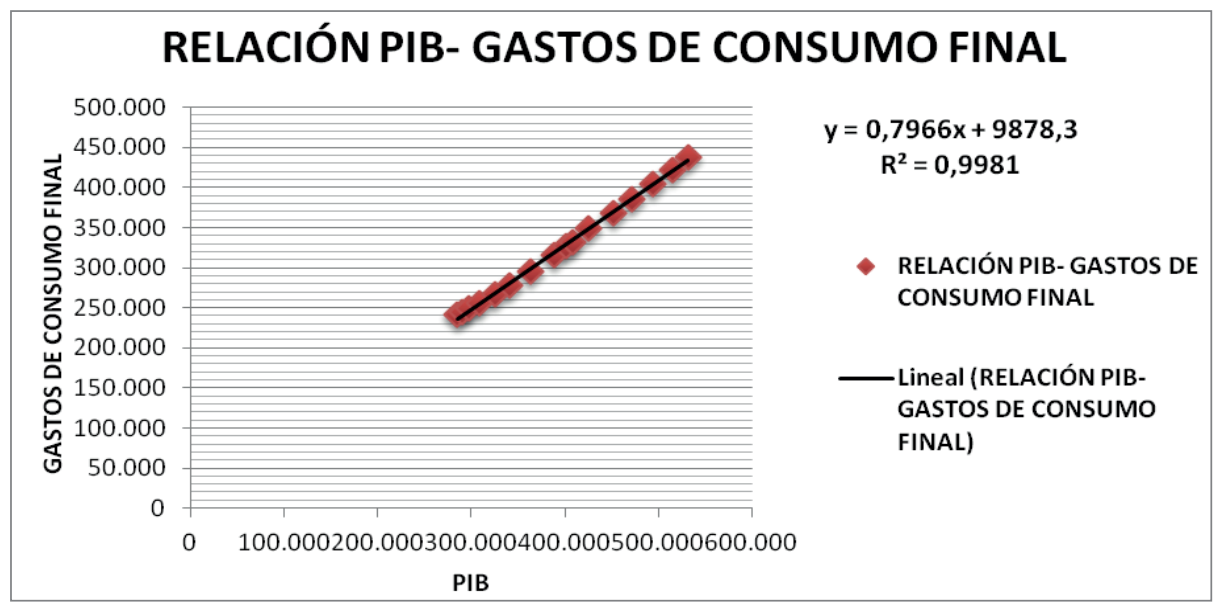

\section{Figura 2}

Correlación lineal ingresos (PIB) y gastos de consumo final colombiano (2000 - 2015).

Fuente: Boletín de Indicadores Económicos 2016, Banco de la República.

En la Fig. 2 Se puede observar que con un 99\%, existe una asociación directa muy alta entre las variables. Es decir, que a medida que aumenta el ingreso (PIB), aumenta significativamente el consumo en Colombia. Así, la $\mathrm{PMgC}$ ha tomado el valor de 0,7966 , lo cual indica que ante un aumento del $1 \%$ en el ingreso (PIB), implicaría un aumento en una menor proporción de un $0,7966 \%$ en el consumo final colombiano, corroborando lo planteado por Morettini (2002).

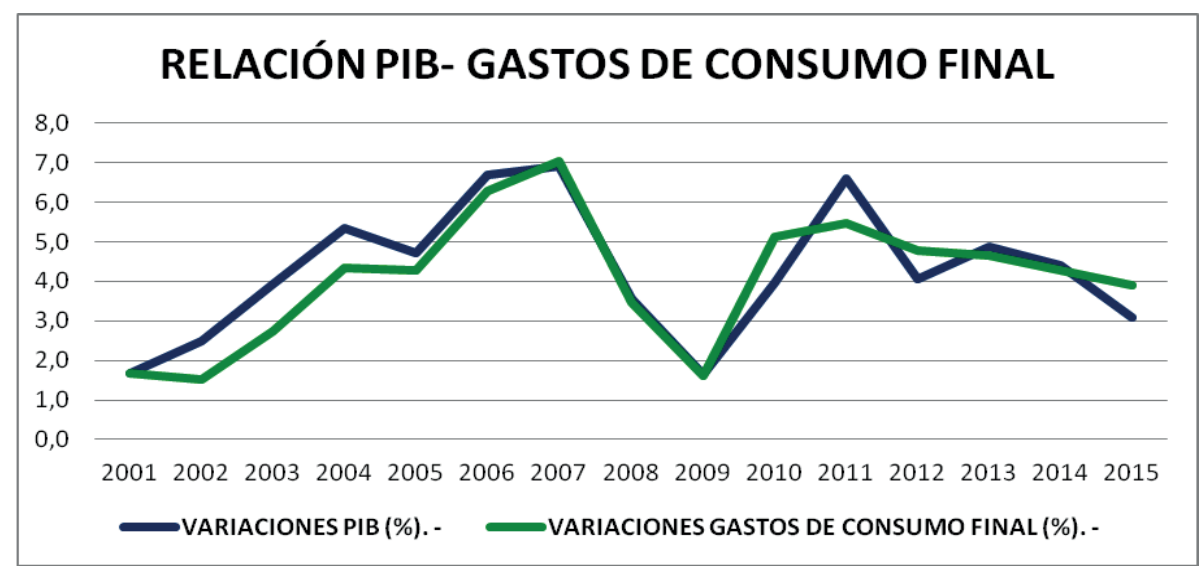

\section{Figura 3}

Variaciones porcentuales ingresos (PIB) y gastos de consumo final colombiano (2001 -2015).

Fuente: Boletín de Indicadores Económicos 2016, Banco de la República. 
En la Fig. 3 Se muestra las variaciones porcentuales de ingreso (PIB) y el consumo final colombiano. El PIB en los años 2006, 2007 y 2011 presentó un incremento significativo y por ende el consumo también creció. Por otro lado, en los años 2002 y 2009 se evidencia una disminución de los ingresos, reduciendo el consumo; con ello se confirma la relación directa entre PIB y consumo en Colombia.

En el gráfico se muestra que en el año 2002 la economía colombiana creció al 2,5\%, esto es explicado, por el aumento en las utilidades y disminución de cartera; en los años 2005 y 2006 la inversión creció a tasas cercanas al 20\%. Durante el año 2006 la economía en Colombia tuvo un crecimiento alrededor del $6,7 \%$, gracias a la inversión y la demanda interna. Esto se presentó desde el año 2005 cuando la cifra fue de $4,7 \%$ debido al dinamismo de sectores como la construcción, transporte, comercio y la industria. De esta manera, en el año 2007, se presentó un descenso en la tasa de cambio y un aumento en las reservas naturales, comparadas con el año anterior.

Como consecuencia de la crisis que presentaron las economías desarrolladas, Colombia experimentó una fuerte desaceleración teniendo así un crecimiento de $1,6 \%$ en el año 2009. Finalmente, los resultados del 2011 en comparación con el año 2010 fueron las variaciones en la explotación de minas y canteras del 14,3\% y en transporte, almacenamiento y comunicaciones del $6,9 \%$ gracias a grandes ramas de actividad. 


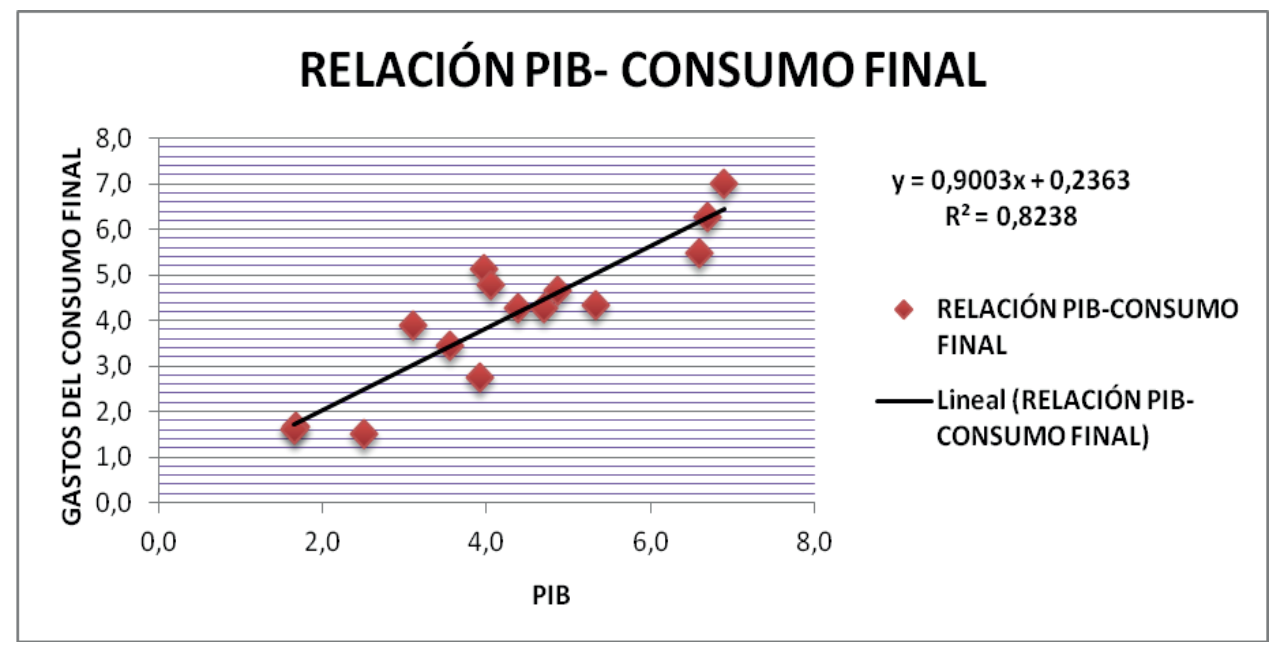

\section{Figura 4}

Correlación porcentual ingresos (PIB) y consumo final en Colombia (2001 - 2015).

Fuente: Boletín de Indicadores Económicos 2016, Banco de la República.

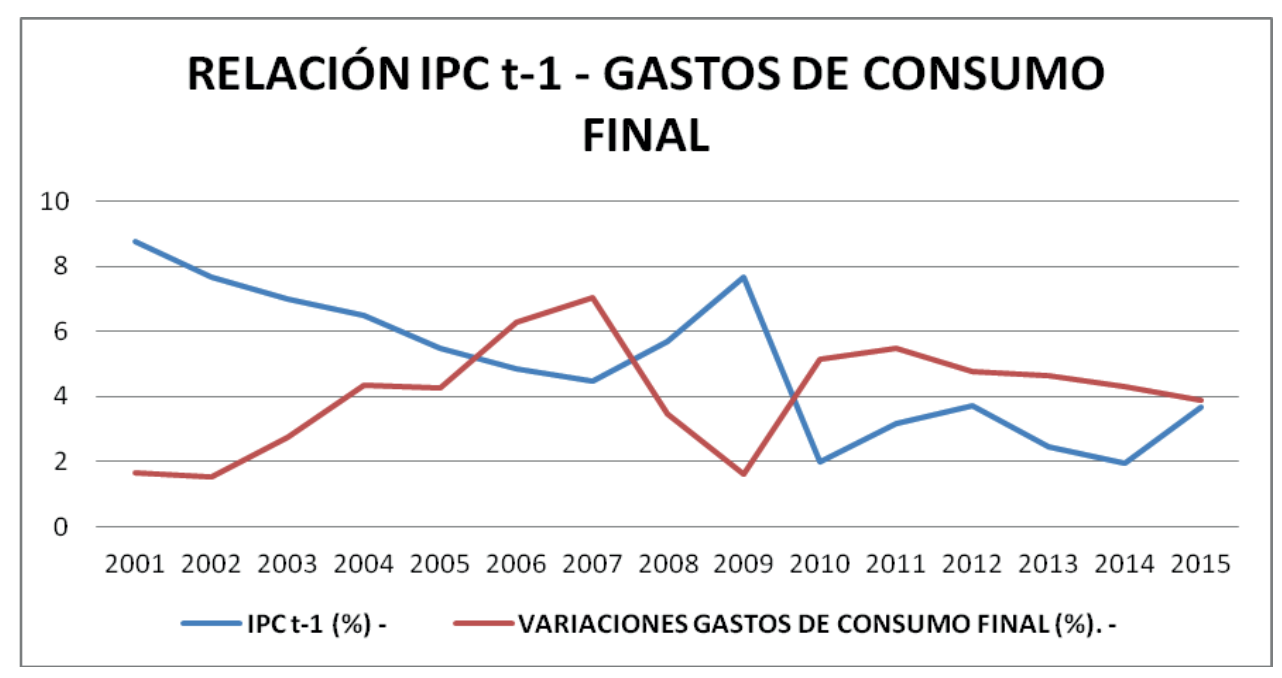

\section{Figura 5}

Variaciones porcentuales IPC t-1 y gastos de consumo final en Colombia (2001 - 2015).

Fuente: Boletín de Indicadores Económicos 2016, Banco de la República.

En la Fig. 5 se presenta la relación IPC t-1 y gastos de consumo final, donde IPC t-1 (Efecto intertemporal) explica el impacto sobre IPC t. Es decir, los efectos en 
el nivel de precios del pasado expresado como IPC t-1 afectan el nivel de precios (IPC) del presente. Esto coincide con lo planteado por Dornbusch, Fischer y Startz (2009), cuando explican que la inflación es la tasa de variación de los precios y, el nivel de precios es determinado por la acumulación de inflaciones anteriores.

En la gráfica se puede apreciar que existe una relación inversa entre los índices de IPC y el consumo en Colombia en el periodo estudiado. Frente a un aumento de la inflación, se presentará una disminución en el consumo según los planteamientos de Dornbusch, Fischer y Startz (2009). Esto se ve reflejado en los años $2001-2004$ y 2009.

Para el año 2009 la inflación desbordó todos los pronósticos, generando la subida de los precios de la canasta familiar de los colombianos, haciendo que haya un aumento del índice de precios al consumidor del 7,67\%, en lo que se observa claramente la relación inversa entre IPC $\mathrm{t}-1$ y consumo, mostrando aumentos del IPC $(6,49 \%-8,75 \%)$, por encima del rango meta impuesto por el Banco de la República ( $2 \%-4 \%$ ), y en el mismo periodo se evidencia una caída fuerte en el consumo del $1,5 \%$ en el 2002.

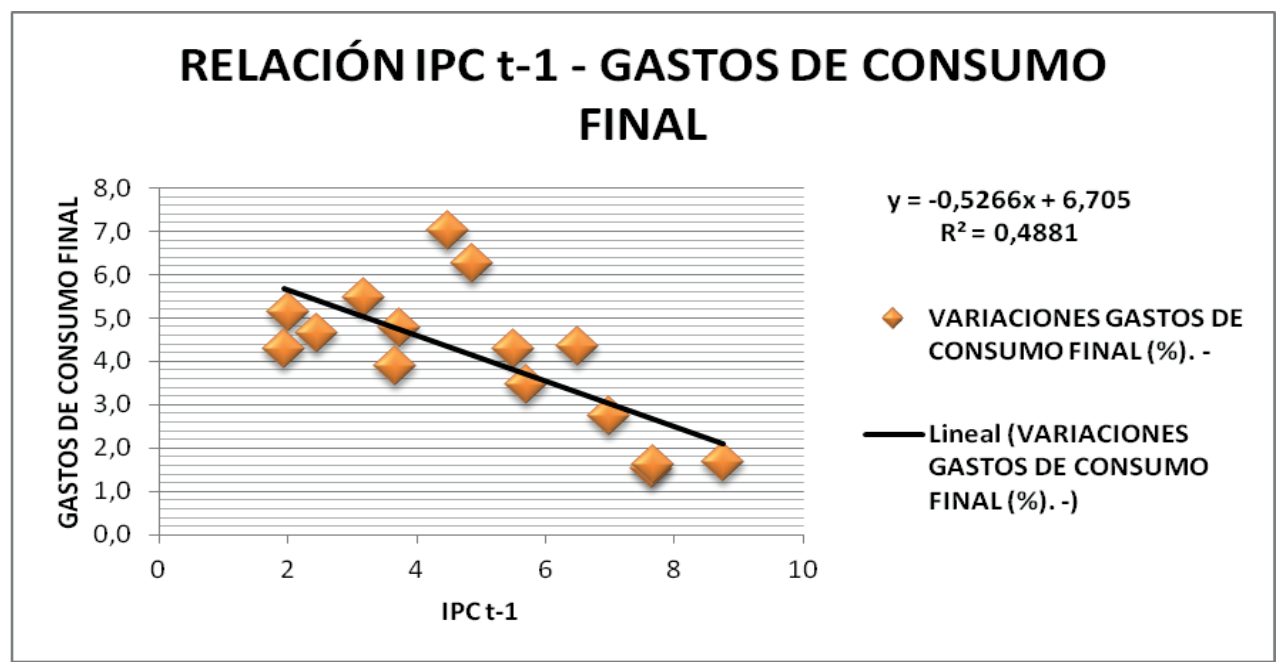

Figura 6

Correlación porcentual IPC t-1 y gastos de consumo final en Colombia (2001 - 2015).

Fuente: Boletín de Indicadores Económicos 2016, Banco de la República. 
La anterior gráfica explica la relación inversa entre IPC t-1 y gastos de consumo final colombiano, lo cual implica que ante el aumento del $1 \%$ en el nivel de precios expresado como IPC t-1, se presentará una disminución de gastos en el consumo de $0,5266 \%$, ya que ante un aumento en el nivel de precios, el consumo se ve afectado negativamente.

Adicionalmente con un 48,81\%, existe una asociación indirecta entre las variables, es decir que a medida que aumenta el IPC $\mathrm{t}$ - 1 , disminuye significativamente el consumo en Colombia.

\section{MODELO DEL CONSUMO COLOMBIANO}

Teniendo en cuenta lo anterior se ha determinado un modelo estadístico que explica el consumo en Colombia:

CONSUMO EN COLOMBIA= f $($ PIB, IPC t-1)

$$
\begin{gathered}
\mathrm{Y}=\beta_{0}+\beta_{1} \mathrm{X}_{1}-\beta_{2} \mathrm{X}_{2}+\mu \\
\mathrm{Y}=2.12936+0.73985 X_{1}-0.241132 X_{2}
\end{gathered}
$$

\section{Dónde:}

Y: Consumo final

及0: Constante

及1X1: Ingreso (PIB)

B2X2: Inflación (IPC t-1)

$\boldsymbol{\mu}$ : Término de error

\section{INTERPRETACIÓN}

1. En Colombia, en el periodo comprendido entre 2000 y 2015, si el PIB se incrementa en $1 \%$, el consumo aumenta en $0,74 \%$; comprobando de esta manera la relación directa planteada en el presente estudio entre los ingresos expresados en las cifras del PIB y el consumo. El PIB explica al consumo con una confianza superior al $95 \%$.

2. Para el mismo periodo de tiempo, el incremento en $1 \%$ de la inflación expresada como IPC t-1 reduce el consumo colombiano en $0,24 \%$. Esta 
variable explica al consumo con un nivel de confiabilidad mayor al 95\% y verifica la relación inversa con el consumo.

3. De acuerdo con el coeficiente de determinación R-cuadrado el consumo en Colombia es explicado con un $90 \%$ de confianza entre las variables PIB e IPC t-1.

\section{CONCLUSIONES}

En Colombia hay dos variables que explican esencialmente el consumo, los ingresos basados en el PIB y el nivel de los precios reflejado en el Índice de precios al Consumidor (IPC).

Esta idea, en razón a que las variables restantes, expuestas en el marco teórico, aunque originan el consumo, no son significativas en el caso colombiano. Así, los impuestos, tasas de interés, desempleo, subsidios y pobreza son causas indirectas del consumo, porque primero inciden en los ingresos disponibles y esto posteriormente determina el consumo. Ocurre una situación similar con variables como la devaluación de la moneda, la cual explica el nivel de precios y las tasas de interés, variables que primero impactan a la inflación, y posteriormente ésta es la que afecta al consumo.

En consecuencia, se recomienda aliviar las cargas tributarias, dinamizar los subsidios y las inversiones en educación contribuyendo a incrementar el ingreso percibido, al tiempo que controlar la tasa de interés para propiciar mejores niveles de consumo en el país, y por supuesto mantener estable y controlada la tasa de inflación, pues como menciona Cano, la inflación es el talón de Aquiles de la economía colombiana y uno de los principales determinantes del consumo en Colombia pues tiene un efecto directo sobre el consumo. 


\section{REFERENCIAS}

(1) Akerlof, G, Dickens, W \& Perry, G. (1996). The Macroeconomics of Low Inflation. enero, 1996, de Brookings Institution and University of California, Berkeley. (En línea). Disponible en: http://www.brookings.edu/ /media/Projects/BPEA/1996-1/1996a bpea akerlof_dickens_perry_gordon_mankiw.PDF

(2) Ando, Albert \& Modigliani, F. (1963) "The Life Cycle Hyphotesis of Saving: Aggegate Implications and Tests”. The American Economic Review, vol. 53, no. 1, march.

(3) Banco de la República (2016). Boletín de Indicadores Económicos 2000 - 2015.

(4) Banco de la República. (2015). Informe de Política Monetaria. abril 3, 2019, de Banco de la República. (En línea). Disponible en: http://www.banrep.gov.co/sites/default/files/ paginas/dmjd mar 2015.pdf

(5) Cano, G. (2007). Reflexión personal del Codirector del Banco de la República Carlos Gustavo Cano sobre el estado de la economía y la moneda. abril 3, 2019, de Banco de la República. (En línea). Disponible en: http://www.banrep.gov.co/docum/Lectura finanzas/ pdf/2006-2007.pdf

(6) Clavijo, S. \& Fernández, J. (1989). Consumo privado e ingreso permanente: Nueva evidencia para Colombia. diciembre, 1989, de Revista ESPE. (En línea). Disponible en: http://www.banrep.gov.co/sites/default/files/publicaciones/archivos/espe_016-1.pdf

(7) Diccionario enciclopédico Quillet. (1972). Finsu Panamá S.A tomo II.

(8) Dornbusch, Rudiger \& Fischer, S. (1993). Macroeconomía. 6º edición. Mc Graw Hill. Madrid.

(9) Dornbusch, R. Fischer, S. \& Startz, R. (2009). Macroeconomía (Novena Edición). Aravaca (Madrid): McGraw-Hill.

(10) Echeverri, R. (1998). Colombia en transición de la crisis a la convivencia: una visión desde lo rural. Colombia: IICA en coedición con TM Editores. 
(11) Friedman, M. (1957), "A Theory of the Consumption Function", Princeton University Press. Existe traducción al español como "Una Teoría de la Función de Consumo" Ed. Alianza Universidad, 1973.

(12) Friedman, M. (1979). Moneda y desarrollo económico (Segunda Edición). Argentina: El Ateneo.

(13) Friedman, M \& Friedman, R. (1988). Libertad de elegir. Barcelona: Ediciones Grijalbo S.A.

(14) Friedman, M. (1993). Teoría de los precios. Barcelona - España: Altaya S.A.

(15) Hernández, J. (2006). Revisión de los determinantes macroeconómicos del consumo total de los hogares para el caso colombiano. diciembre, 2006, de Revista ESPE. (En línea). Disponible en: http://www.banrep.gov.co/sites/default/files/publicaciones/archivos/ espe 052-2.pdf

(16) Iregui, A. \& Melo, L. (2009). La transmisión de la política monetaria sobre el consumo en presencia de restricciones de liquidez. abril, 2009, de Banco de la República. (En línea). Disponible en: http:/www.banrep.gov.co/sites/default/files/publicaciones/archivos/119. $\underline{\mathrm{pdf}}$

(17) Jiménez, F. (2006). Macroeconomía Enfoques y Modelos (Tomo I), Tercera Edición. Lima - Perú: Fondo Editorial de la Pontificia Universidad Católica del Perú.

(18) Keynes, J. M. (1936). Teoría general de la ocupación, el interés y el dinero. Fondo de Cultura Económica. México, 1951.

(19) Morishima, M. (1981). Teoría económica de la sociedad moderna. Barcelona, España: Antoni Bosch Editor.

(20) Keynes, J. (1983). La teoría general de la ocupación, el interés y el dinero, México: Fondo de Cultura Económica, décima primera reimpresión, 1983.

(21) Keynes, J. (1992). Teoría general de la ocupación, el interés y el dinero; $2^{\text {o }}$ edición, pp. 93, Fondo de cultura económica. Buenos Aires.

(22) León, C \& Miranda, M. (2003). Análisis Macroeconómico para la Empresa. abril 3, 2019, de Universidad Católica Santo Toribio de Mogrovejo. (En línea). Disponible en: http:// www.eumed.net/cursecon/libreria/cl-mm-macro/cl-mm-macro.pdf 
(23) Modigliani, F. (1949) "Fluctuations in the Saving-income Ratio: A Problem in Economic Forecasting", Studies in Income and Wealth, vol. 11, parte V, pp. 369-444, National Bureau of Economic Research, 1949.

(24) Modigliani, F. (1986). Life cycle, individual trift, and the wealth of nations. American economic review, 7, 297-313.

(25) CEPAL. (2001). Pobreza. Definición conceptual. julio 7, 2019, de Comisión Económica para América Latina y el Caribe (CEPAL) Sitio web: http://catarina.udlap.mx/u_dl_a/tales/ documentos/lec/escaroz_c_ga/capitulo1.pdf

(26) Morettini, M. (2002). Principales teorías macroeconómicas sobre el Consumo. Mayo, 2002, de Universidad Nacional de Mar del Plata. (En línea). Disponible en: http://nulan. mdp.edu.ar/1887/1/01486.pdf

(27) Morten O. Ravn. (2008).The Consumption-Tightness Puzzle. July, 2008, de University of Chicago Press. (En línea). Disponible en: http://www.nber.org/chapters/c7034

(28) Otálora, C. (2009). Economía fiscal. La paz, Bolivia: Plural Editores. 


\section{ANEXOS}

Anexo. 1 Base de datos utilizada en el estudio.

\begin{tabular}{|c|c|c|c|c|c|c|}
\hline $\mathbf{A N ̃ O}$ & $\begin{array}{c}\text { CONSUMO } \\
\text { FINAL (\%) }\end{array}$ & $\begin{array}{c}\text { INGRESO } \\
\text { (PIB) \% }\end{array}$ & IPC (\%) & IPC t-1 (\%) & $\begin{array}{c}\text { DEVALUACIÓN } \\
\text { NOMINAL (\%) }\end{array}$ & $\begin{array}{c}\text { DEVALUACIÓN } \\
\text { REAL (\%) }\end{array}$ \\
\hline $\mathbf{2 0 0 0}$ & - & - & 8,75 & - & 18,97 & 6,76 \\
\hline $\mathbf{2 0 0 1}$ & 1,7 & 1,7 & 7,65 & 8,75 & 2,18 & $-5,08$ \\
\hline $\mathbf{2 0 0 2}$ & 1,5 & 2,5 & 6,99 & 7,65 & 25,04 & 13,73 \\
\hline $\mathbf{2 0 0 3}$ & 2,7 & 3,9 & 6,49 & 6,99 & $-3,02$ & 4,27 \\
\hline $\mathbf{2 0 0 4}$ & 4,3 & 5,3 & 5,5 & 6,49 & $-13,98$ & $-10,88$ \\
\hline $\mathbf{2 0 0 5}$ & 4,3 & 4,7 & 4,85 & 5,5 & $-4,42$ & $-2,52$ \\
\hline $\mathbf{2 0 0 6}$ & 6,3 & 6,7 & 4,48 & 4,85 & $-1,99$ & 0,12 \\
\hline $\mathbf{2 0 0 7}$ & 7,0 & 6,9 & 5,69 & 4,48 & $-10,01$ & $-0,67$ \\
\hline $\mathbf{2 0 0 8}$ & 3,5 & 3,5 & 7,67 & 5,69 & 11,36 & $-2,70$ \\
\hline $\mathbf{2 0 0 9}$ & 1,6 & 1,7 & 2 & 7,67 & $-8,89$ & $-2,79$ \\
\hline $\mathbf{2 0 1 0}$ & 5,1 & 4,0 & 3,17 & 2 & $-6,37$ & $-3,93$ \\
\hline $\mathbf{2 0 1 1}$ & 5,5 & 6,6 & 3,73 & 3,17 & 1,50 & $-1,08$ \\
\hline $\mathbf{2 0 1 2}$ & 4,8 & 4,0 & 2,44 & 3,73 & $-8,98$ & $-2,74$ \\
\hline $\mathbf{2 0 1 3}$ & 4,6 & 4,9 & 1,94 & 2,44 & 8,97 & 6,17 \\
\hline $\mathbf{2 0 1 4}$ & 4,3 & 4,4 & 3,66 & 1,94 & 24,17 & 7,67 \\
\hline $\mathbf{2 0 1 5}$ & 3,9 & 3,1 & 6,77 & 3,66 & 31,64 & 13,94 \\
\hline
\end{tabular}

Fuente: Boletín de Indicadores Económicos 2016, Banco de la República

\section{Anexo. 2 Base de datos utilizada en el estudio}

\begin{tabular}{|c|c|c|}
\hline AÑO & $\begin{array}{c}\text { PIB (Miles de millones } \\
\text { de pesos). }\end{array}$ & $\begin{array}{c}\text { GASTOS DE CONSUMO FINAL (Miles de } \\
\text { millones de pesos). }\end{array}$ \\
\hline $\mathbf{2 0 0 0}$ & 284.761 & 240.964 \\
\hline $\mathbf{2 0 0 1}$ & 289.539 & 245.000 \\
\hline $\mathbf{2 0 0 2}$ & 296.789 & 248.703 \\
\hline $\mathbf{2 0 0 3}$ & 308.418 & 255.501 \\
\hline $\mathbf{2 0 0 4}$ & 324.866 & 266.562 \\
\hline $\mathbf{2 0 0 5}$ & 340.156 & 277.953 \\
\hline $\mathbf{2 0 0 6}$ & 362.938 & 295.370 \\
\hline $\mathbf{2 0 0 7}$ & 387.983 & 316.105 \\
\hline $\mathbf{2 0 0 8}$ & 401.744 & 327.011 \\
\hline $\mathbf{2 0 0 9}$ & 408.379 & 332.275 \\
\hline $\mathbf{2 0 1 0}$ & 424.599 & 349.303 \\
\hline $\mathbf{2 0 1 1}$ & 452.578 & 368.399 \\
\hline $\mathbf{2 0 1 2}$ & 470.880 & 386.023 \\
\hline $\mathbf{2 0 1 3}$ & 493.831 & 403.945 \\
\hline $\mathbf{2 0 1 4}$ & 515.489 & 421.269 \\
\hline $\mathbf{2 0 1 5}$ & 531.376 & 437.797 \\
\hline
\end{tabular}

Fuente: Boletín de Indicadores Económicos 2016, Banco de la República 
Principales determinantes económicos del consumo en Colombia 2000 - 2015

Cabrera Valverde _ Diana Gisell, Rosero Oliveros _ Tania Marcela, Riascos _ Julio César

\section{Anexo. 3 Análisis del Modelo estadístico en Gretl}

\section{- MODELO 1:}

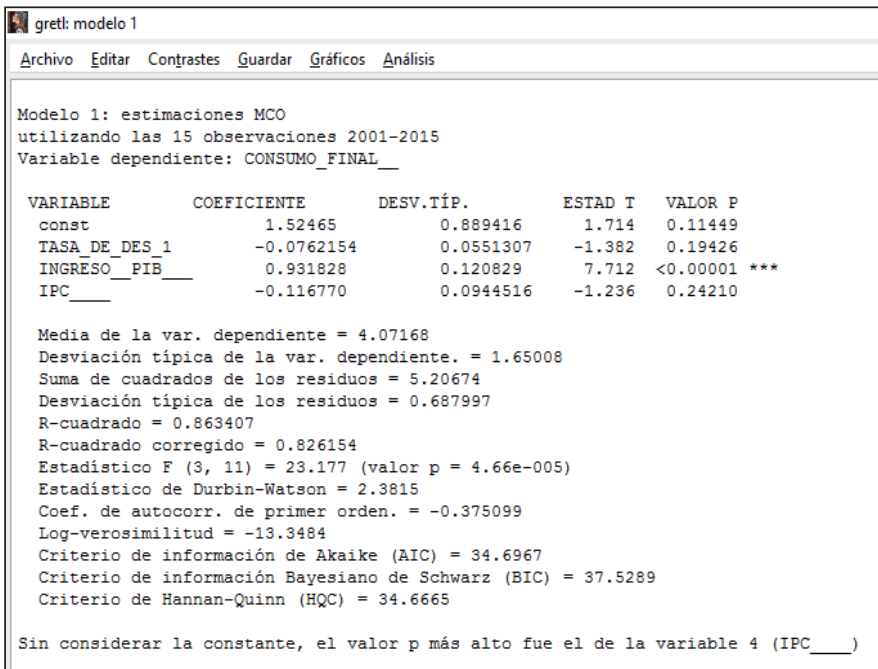

\begin{tabular}{|c|c|c|c|}
\cline { 2 - 4 } \multicolumn{1}{c|}{} & PIB & IPC & DESMPLEO t-1 \\
\hline valor $\mathbf{P}$ & 0,00001 & 0,2421 & 0,19426 \\
\hline \multirow{4}{*}{} & 0,000005 & 0,12105 & 0,09713 \\
\cline { 2 - 4 } \multicolumn{1}{c|}{} & $\mathbf{1 0 0} \%$ & $\mathbf{8 8 \%}$ & $\mathbf{9 0 \%}$ \\
\hline R-cuadrado & \multicolumn{3}{|c|}{$86 \%$} \\
\hline
\end{tabular}

\section{- MODELO 2:}

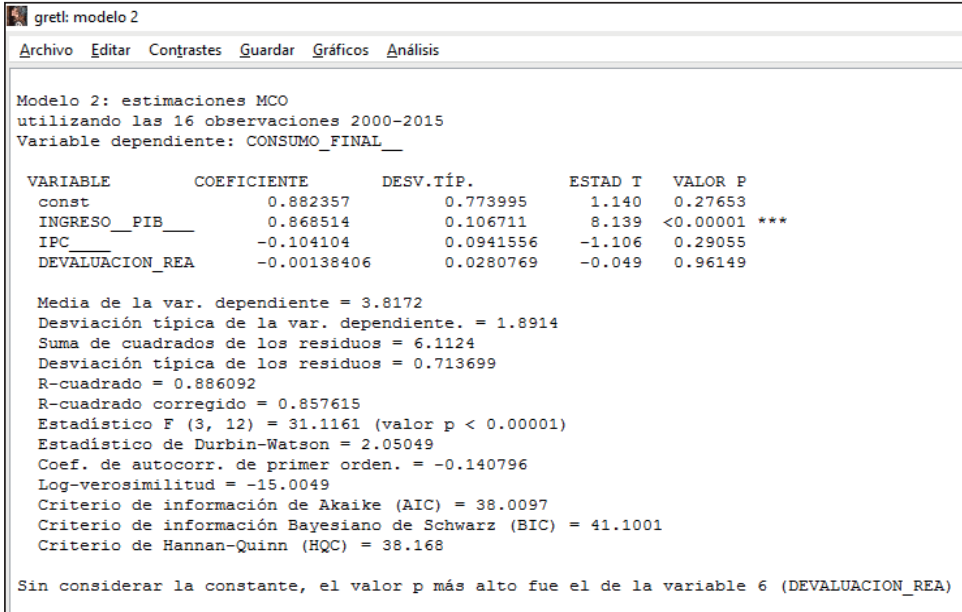




\begin{tabular}{|c|c|c|c|}
\cline { 2 - 5 } \multicolumn{1}{c|}{} & PIB & IPC & Devaluación real \\
\hline valor $\mathbf{P}$ & 0,00001 & 0,29055 & 0,96149 \\
\hline \multirow{4}{*}{} & 0,000005 & 0,145275 & 0,480745 \\
\cline { 2 - 4 } & $\mathbf{1 0 0 \%}$ & $\mathbf{8 5 \%}$ & $\mathbf{5 2 \%}$ \\
\hline R-cuadrado & \multicolumn{3}{|c|}{$89 \%$} \\
\hline
\end{tabular}

- MODELO 3:

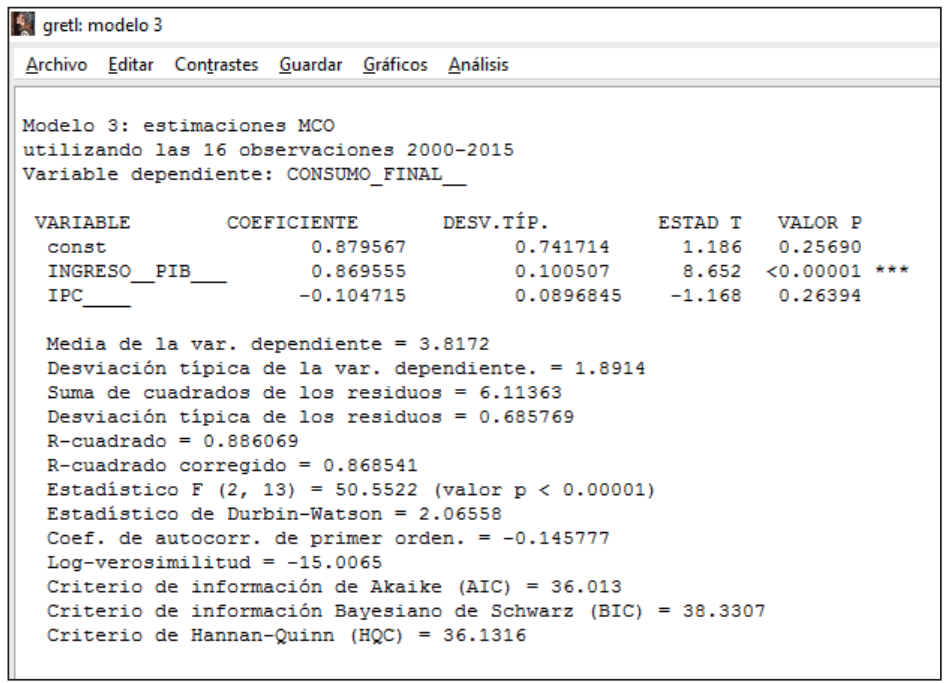

\begin{tabular}{|c|c|c|}
\cline { 2 - 3 } \multicolumn{1}{c|}{} & PIB & IPC \\
\hline valor $\mathbf{P}$ & 0,00001 & 0,26394 \\
\hline \multirow{4}{*}{0,000005} & 0,13197 \\
\cline { 2 - 3 } & $\mathbf{1 0 0} \%$ & $\mathbf{8 7 \%}$ \\
\hline
\end{tabular}

\begin{tabular}{|l|c|}
\hline R-cuadrado & $89 \%$ \\
\hline
\end{tabular}


Principales determinantes económicos del consumo en Colombia 2000 - 2015

Cabrera Valverde _ Diana Gisell, Rosero Oliveros _ Tania Marcela, Riascos _ Julio César

\section{- MODELO 4:}

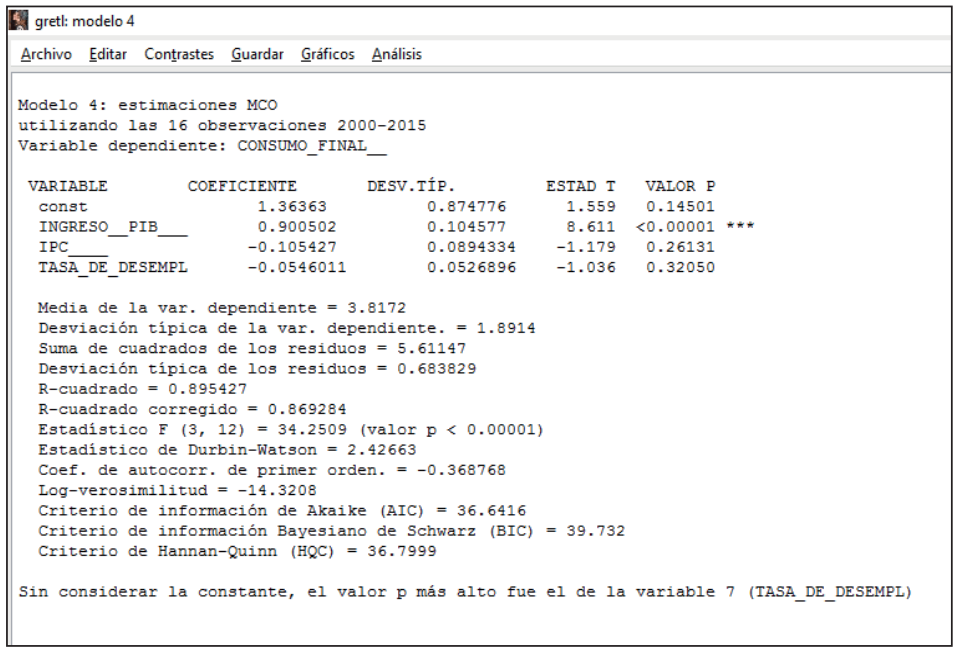

\begin{tabular}{|c|c|c|c|}
\cline { 2 - 4 } \multicolumn{1}{c|}{} & PIB & IPC & DESEMPLEO \\
\hline \multirow{2}{*}{ valor $\mathbf{P}$} & 0,00001 & 0,26131 & 0,3205 \\
\hline & 0,000005 & 0,130655 & 0,16025 \\
\cline { 2 - 4 } & $\mathbf{1 0 0 \%}$ & $\mathbf{8 7 \%}$ & $\mathbf{8 4 \%}$ \\
\hline R-cuadrado & \multicolumn{3}{|c|}{$90 \%$} \\
\hline
\end{tabular}

\section{- MODELO 5:}

\begin{tabular}{|c|c|c|c|c|c|}
\hline \multicolumn{6}{|c|}{$\begin{array}{c}\text { Modelo 5: estimaciones MCO utilizando las } 15 \text { observaciones 2001-2015 } \\
\text { Variable dependiente: CONSUMO_FINAL_ }\end{array}$} \\
\hline Variable & Coeficiente & Desv. típica & Estadístico $t$ & valor $p$ & \\
\hline const & 0.788125 & 0.674793 & 1.1680 & 0.26551 & \\
\hline INGRESO_PIB_ & 0.957351 & 0.121645 & 7.8701 & $<0.00001$ & $* * *$ \\
\hline $\begin{array}{l}\text { TASA_DE_DES_ } \\
1\end{array}$ & -0.070926 & 0.0561615 & -1.2629 & 0.23062 & \\
\hline \multicolumn{6}{|c|}{ Media de la var. dependiente $=4.07168$} \\
\hline \multicolumn{6}{|c|}{ Desviación típica de la var. dependiente. $=1.65008$} \\
\hline \multicolumn{6}{|c|}{ Suma de cuadrados de los residuos $=5.9302$} \\
\hline \multicolumn{6}{|c|}{ Desviación típica de los residuos $=0.702981$} \\
\hline \multicolumn{6}{|c|}{$\mathrm{R}^{2}=0.844428$} \\
\hline \multicolumn{6}{|c|}{$\mathrm{R}^{2}$ corregido $=0.818499$} \\
\hline \multicolumn{6}{|c|}{ Estadístico F $(2,12)=32.5673($ valor $\mathrm{p}=1.42 \mathrm{e}-005)$} \\
\hline \multicolumn{6}{|c|}{ Estadistico de Durbin-Watson $=1.98549$} \\
\hline \multicolumn{6}{|c|}{ Coef. de autocorr. de primer orden $=-0.0994005$} \\
\hline \multicolumn{6}{|c|}{ Log-verosimilitud $=-14.3241$} \\
\hline \multicolumn{6}{|c|}{ Criterio de información de Akaike $=34.6483$} \\
\hline \multicolumn{6}{|c|}{ Criterio de información Bayesiano de Schwarz $=36.7724$} \\
\hline \multicolumn{6}{|c|}{ Criterio de Hannan-Quinn $=34.6256$} \\
\hline
\end{tabular}




\section{- MODELO 6:}

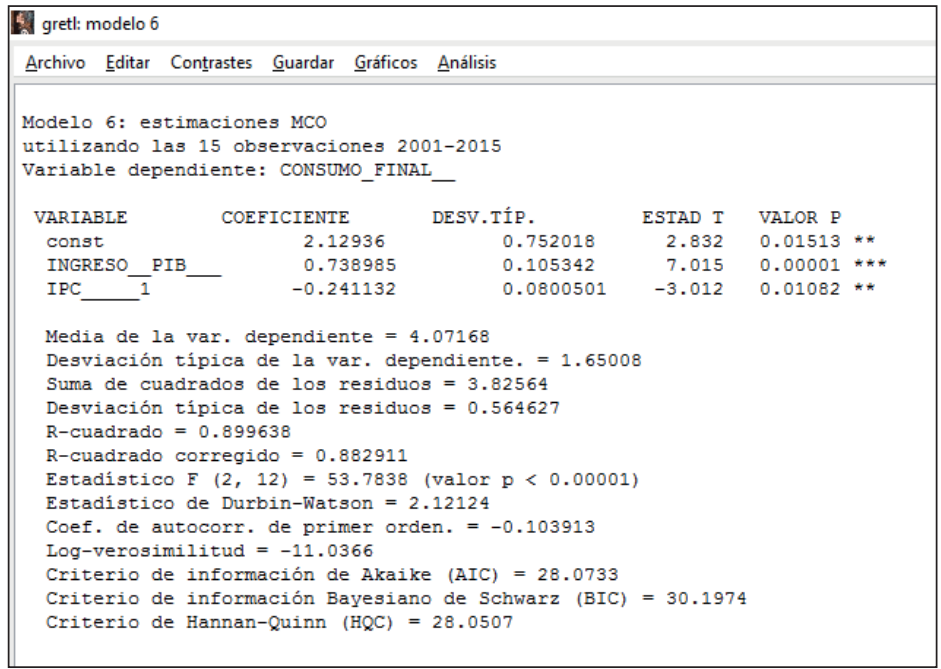

\begin{tabular}{|c|c|c|}
\cline { 2 - 3 } \multicolumn{1}{c|}{ MODELO 6 } & \multicolumn{2}{c|}{ MODELO SELECCIONADO } \\
\cline { 2 - 3 } \multicolumn{1}{c|}{ valor P } & 0,00001 & IPC t- 1 \\
\hline \multirow{4}{*}{} & 0,000005 & 0,01082 \\
\cline { 2 - 3 } & $\mathbf{1 0 0 \%}$ & 0,00541 \\
\cline { 2 - 3 } & & $\mathbf{9 9} \%$ \\
\hline
\end{tabular}

\begin{tabular}{l|l} 
R-cuadrado & $90 \%$
\end{tabular}

\section{- MODELO 7:}

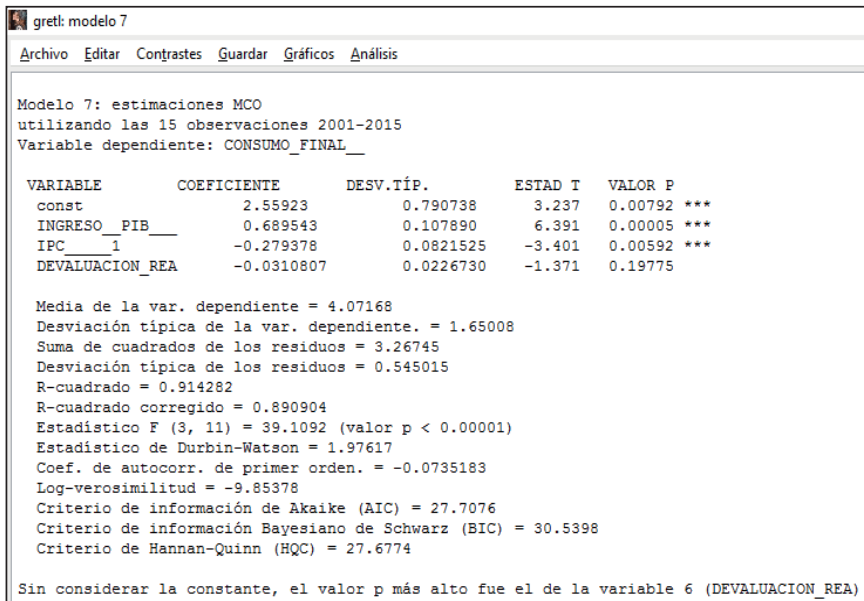

Sin considerar la constante, el valor p más alto fue el de la variable 6 (DEVALUACION REA) 
Principales determinantes económicos del consumo en Colombia 2000 - 2015 Cabrera Valverde _ Diana Gisell, Rosero Oliveros _ Tania Marcela, Riascos _ Julio César

\begin{tabular}{|c|c|c|c|}
\cline { 2 - 4 } \multicolumn{1}{c|}{} & PIB & IPC t-1 & Devaluación real \\
\hline \multirow{2}{*}{ valor P } & 0,00005 & 0,00592 & 0,19775 \\
\hline & 0,000025 & 0,00296 & 0,098875 \\
\cline { 2 - 4 } \multicolumn{1}{c|}{} & $\mathbf{1 0 0 \%}$ & $\mathbf{1 0 0 \%}$ & $\mathbf{9 0 \%}$ \\
\hline R-cuadrado & \multicolumn{3}{|c|}{$91 \%$} \\
\hline
\end{tabular}

\section{- MODELO 8:}

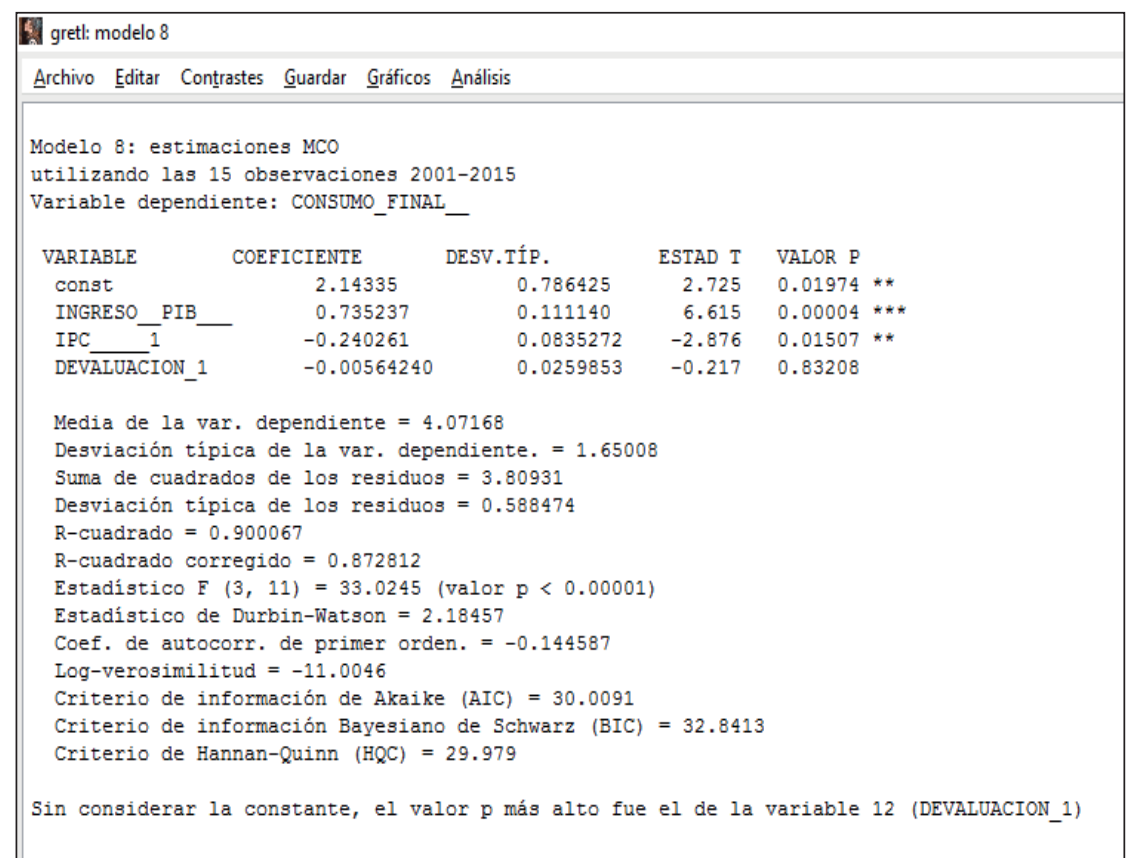

\begin{tabular}{|c|c|c|c|}
\cline { 2 - 4 } \multicolumn{1}{c|}{} & PIB & IPC t-1 & Devaluación real t-1 \\
\hline valor $\mathbf{P}$ & 0,00004 & 0,01507 & 0,83208 \\
\hline & 0,00002 & 0,007535 & 0,41604 \\
\cline { 2 - 4 } & $\mathbf{1 0 0 \%}$ & $\mathbf{9 9 \%}$ & $\mathbf{5 8 \%}$ \\
\hline R-cuadrado & \multicolumn{3}{|c|}{$90 \%$} \\
\hline
\end{tabular}

Fuente: elaboración propia. 